\title{
Battery revolution to evolution
}

\author{
The revolutionary work of John Goodenough, M. Stanley Whittingham and Akira Yoshino has finally been awarded \\ the Nobel Prize in Chemistry. Scientific discovery and engineering brilliance continue to shape battery technology.
}

S o much has been said about the astonishing advancements of and societal transformations brought about by Li-ion batteries (LIBs) in portable electronics, and more recently transportation and grid-scale storage, that the recognition from the 2019 Nobel committee to the three LIB pioneers in October feels long overdue. Nonetheless, the story of their seminal work in laying the foundations of Li-ion chemistry bears repeating. At the same time, it is important to recognize the key milestones along the road to today's commercial LIBs and to consider the path ahead to the batteries of the future.

The revolution started during the oil crisis of the 1970s when society was hungering for alternative energy sources to replace fossil fuels. Batteries then, such as lead-acid and nickel-cadmium, did not offer much hope for high-energy output. Writing first in the Journal of the Chemical Society, Chemical Communications in $1974^{1}$, M. Stanley Whittingham noted that ions can be electrochemically intercalated into layered transition-metal disulfides such as $\mathrm{TiS}_{2}$. This intercalation chemistry, as he demonstrated subsequently in Science in 1976, enabled the first rechargeable $\mathrm{Li}$ battery, which consisted of a $\mathrm{TiS}_{2}$ cathode and a metallic Li anode ${ }^{2}$.

Writing in the Materials Research Bulletin in $1980^{3}$, John Goodenough and co-workers reported a cathode, $\mathrm{LiCoO}_{2}$ (LCO), that has a similar layered structure to $\mathrm{TiS}_{2}$ and is also capable of (de)intercalating $\mathrm{Li}$ ions. However, this new cathode doubled the operating voltage of $\mathrm{TiS}_{2}$ and thus led to a significantly higher energy density. Among the many cathode materials, LCO is the most successful for portable devices: it is still being used in the majority of smart phones today.

Early rechargeable Li batteries were only successful in the lab. A main problem lies in the use of metallic Li based anodes, which have high chemical activity leading to significant side reactions. They also tend to form dendrites because of Li-ion plating during charging, which causes the hazard of short-circuiting. This was also one of the scientific reasons behind Exxon's unsuccessful attempt to commercialize Whittingham's $\mathrm{Li}_{-} \mathrm{TiS}_{2}$ system. The breakthrough came with the arrival of a 1985 patent $^{4}$, in which Akira Yoshino and colleagues reported the very first practical $\mathrm{Li}$-ion prototype, presenting carbonaceous materials as the anode and LCO as the cathode in a non-aqueous electrolyte.

While Yoshino's cell may appear incredibly simple nowadays, it opened up a new design concept: the reactive metallic Li anode could be replaced by much more benign non-metal compounds such as carbonaceous materials, which are capable of storing Li ions while eliminating problems associated with the use of metallic Li. Sony Corporation soon adopted Yoshino's strategy and made the world's first commercial LIBs with a soft-carbon anode and a LCO cathode, achieving an energy density of $80 \mathrm{Wh} \mathrm{kg}^{-1}$, double that of a typical lead-acid battery. The company later adapted a graphite anode and a polymer gel electrolyte to produce the first commercial polymer batteries. The move to graphite was important as it allowed higher operating voltages, which, along with an optimized LCO cathode, produced an energy density of $190 \mathrm{Wh} \mathrm{kg}^{-1}$. The electrolyte innovation also led to a significant cost reduction as well as safety improvement ${ }^{5}$.

While portable electronics have successfully been powered by graphite-LCO batteries, vehicle electrification craves for much more energy-dense power sources. There have been enormous efforts in searching for future electrodes to enable this. Among the most promising approaches, metallic Li offers the largest specific capacity of all Li-based anodes, despite facing the same problematic plating issues that once halted commercial efforts in the 1970s. However, recent strategies (for example, with the aid of nanotechnology and advancements in electrolyte development) have been able to tame these problems, signalling a revival of the once-popular anode. While it is still early days for practical applications, writing in Nature Energy, Jun Liu, along with Goodenough and Whittingham in their first jointly-authored paper, presents distinct pathways for realizing high-energy long-cycling Li metal batteries $^{6}$. It is also particularly encouraging that a practical prototype Li metal battery is now capable of delivering $300 \mathrm{Wh} \mathrm{kg}^{-1}$ with stable long cycles ${ }^{7}$.

On the cathode front, layered $\mathrm{Ni}-\mathrm{Mn}-\mathrm{Co}$ oxides and $\mathrm{Ni}-\mathrm{Co}-\mathrm{Al}$ oxides are among the most important in today's electric automobiles. These materials bear a close similarity to their ancestor, LCO, but greatly benefit from adjustable compositions and properties from their elemental components. Intense research efforts are currently underway for next generation cathodes such as layered Li- and Mn-rich oxides, and Ni-rich oxides, which could offer higher voltages or capacities and thus higher energy densities.

The goals of future battery discovery remain the same as those faced by the LIB pioneers: more energy and power, longer cycles, lower costs and greater safety. As with the previous scientific breakthroughs coupled with engineering brilliance that enabled the leap forward for battery technologies in the past, advances on both the fundamental and technological levels are still key for the forever evolving batteries of tomorrow. With the increasing interest and considerable investment that is happening around the globe, we look forward to an even brighter electrified future.

Published online: 14 November 2019 https://doi.org/10.1038/s41560-019-0503-2

\footnotetext{
References

1. Whittingham, M. S. J. Chem. Soc. Chem. Commun. 328-329 (1974)

2. Whittingham, M. S. Science 192, 1126-1127 (1976),

3. Mizushima, K., Jones, P. C., Wiseman, P. J. \& Goodenough, J. B. Mater. Res. Bull. 15, 783-798 (1980).

4. Yoshino, A., Sanechikat, K. \& Nakajima, T. Secondary battery. US patent 4,668,595 (1985).

5. Nishi, Y. J. Power Sources 100, 101-106 (2001).

6. Liu, J. et al. Nat. Energy 4, 180-186 (2019).

7. Niu, C. et al. Nat. Energy 4, 551-559 (2019).
} 